\title{
Performance Analysis of Current Ratio under Different Dielectric Constant for Carbon Nanotube Field Effect Transistor
}

\author{
Md. Moshiur Rahman ${ }^{1}$, Md. Motiur Rahman Sagar ${ }^{1}$, Shafkat Tasnim", \\ Mohammad Farhan ${ }^{1}$, MD. Tahmidur Rahman ${ }^{1}$ \\ Department of EEE, Ahsanullah University of Science and Technology, Dhaka, Bangladesh ${ }^{1}$
}

\begin{abstract}
This paper represents carbon nanotube field effect transistors (CNTFETs) under ballistic condition with FETTOY Model based on the changes of gate oxide thickness and tube diameter, the performance has been observed as a function of dielectric constant of oxide material. Details observation of the combined ballistic effect on the basis of the performance of CNTFETs has been emphasized and the outcome of the device has been explored. Throughout the process, the effect of gate oxide material on the ON-state to OFF-state current ratio has been analyzed considering tube diameter and oxide thickness as constants and it is observed that after a certain value of the dielectric constant of oxide material the current ratio remains unchanged. Gate oxide thickness and tube diameter has also been varied to determine their effect on the ON-state to OFF-state current ratio which also indicates a range of diameter and oxide thickness to maintain unchanged current ratio.
\end{abstract}

Keywords: Current ratio, Gate oxide, Tube diameter, Dielectric constant, Gate control parameter.

\section{INTRODUCTION}

Carbon nanotube field effect transistor (CNTFET) has become an efficient field of application of carbon nanotube because of its superior quality like higher ONstate current, high channel density and high electric density [1,2]. In fact such kinds of qualities make CNTFET way better than MOSFET though it dispenses electrons from source terminal to drain terminal just like MOSFET [3-6]. One most important feature of CNTFET is their 1dimentional characteristics because of that the carriers are confined in the carbon nanotube(CNT) and this results rise to a strong quantization of carriers states and charge transport becomes possible in room temperature in the 1D sub-band of CNT. As the space for phase scattering event is reduced this results the probability of back scattering is reduced and enhanced a high conductivity of CNT.[10].

There is another advantage of using CNT is that it not bounded to use silicon oxide as an insulator because all chemical bonds of Carbon in the nanotube is satisfied and there is no need of dangling bond as in Silicon this results the flexibility of using any high dielectric material as an insulator. The use of high dielectric material considering other things a CNTFET can be fabricated which gives CNTs high mechanical and thermal stability and resistance to electromigration. A CNT transistor shows ballistic nature due to smaller channel length but the larger coulomb blockade length and it exhibits non ballistic condition because of energy variance [7]. And the mobility of carrier changes as the outcome of oscillations of the transmission co-efficient of carrier which helps to travel a single defect coulomb potential channel. An inverse relationship between gate oxide thickness and drain current is seen in CNTFET as well as because of elastic scattering channel resistance increases which has an impact on drain current. There is an impressive change in band gap and strain effect plays a vital role in this case [8]. When it is non-ballistic, tunnel current is the highly responsible element.

In this paper, the effects of ON-state to OFF-state current ratio of gate oxide material as well as their characteristics has been analyzed, depending on different component like tube diameter, oxide thickness, gate control parameter, drain control parameter and all the results stand for different dielectric materials of non-ballistic arrangement.

\section{MATHEMATICAL MODEL}

In this section the FETTOY model of CNTFET has been discussed which is original MOSFET-like structure that could achieve near ballistic transport.[9]. However this model can also be used for non-ballistic condition [8]. For the purpose of investigation this model has been analysed based on the different parametric variation of CNTFET. For a specified range of drain/gate voltage the total charge approximation is made to calculate the drain current occupied by the first sub-band in the CNT. Due to this electric field between the drain and the source a nonequilibrium mobile charge is made can be expressed as

$$
\Delta Q=q\left(N_{s}+N_{D}-N_{0}\right)
$$

Where,

$N_{s}$ is the density of positive velocity states filled by the source,

$N_{D}$ is the density of negative velocity states filled by the drain and

$N_{0}$ is the equilibrium electron density. 


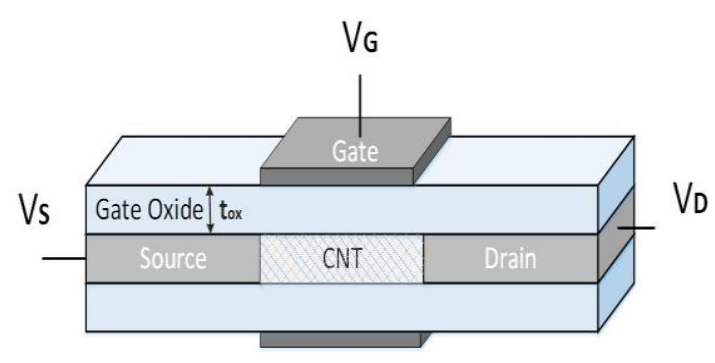

Fig 1(a). Device structure of CNTFET.

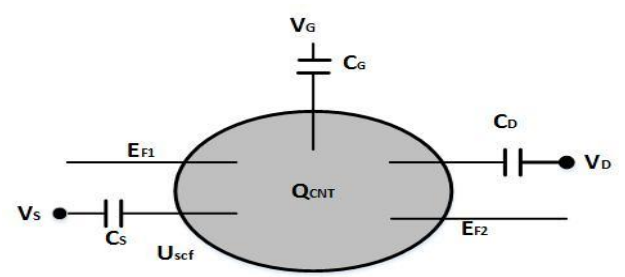

Fig 1(b). 2D capacitor model of CNTFET[9].

To determine the mobile charge the self-consistent potential $U_{s c f}$ needs to be determined which involves solving the two dimensional Poisson equation by using superposition. So Laplace potential due to terminal biases at the top barrier can be expressed as [9]

$$
\mathrm{U}_{\mathrm{L}}=-q\left(\alpha_{G} V_{G}+\alpha_{D} V_{D}+\alpha_{S} V_{S}\right)
$$

In equation (2) $V_{G}$ is the gate voltage, $V_{D}$ is the drain voltage and $V_{S}$ and is the source voltage,

$$
\alpha_{G}=\frac{C_{G}}{C_{\Sigma}}, \alpha_{D}=\frac{C_{D}}{C_{\Sigma}}, \alpha_{S}=\frac{C_{S}}{C_{\Sigma}}
$$

These three $\alpha$ describes how the gate, drain and source control the Laplace solution. Here $C_{\Sigma}$ is the parallel combination of the three capacitors as shown in Fig. 1(b).

$$
U_{P}=\frac{q^{2}}{C_{\Sigma}} \Delta N
$$

So the self -consistent potential at the top of the barrier can be written as

$$
\begin{gathered}
U_{s c f}=U_{L}+U_{P} \\
U_{s c f}=-q\left(\alpha_{G} V_{G}+\alpha_{D} V_{D}+\alpha_{S} V_{S}\right)+\frac{q^{2}}{C_{\Sigma}} \Delta N
\end{gathered}
$$

As the mobile current is determined by the local density of state at the top of the barrier, location of the source and drain levels, $E_{f 1}$ and $E_{f 2}$ and self-consistent potential at the top the barrier, $U_{s c f}$

So finally the drain current can be found by the equation as follows [9]

$$
I_{D}=\frac{2 q k_{B} T}{\hbar}\left[\ln \left(1+e^{\left(E_{f 1}-U_{s c f}\right)}\right)-\ln \left(1+e^{\left(E_{f 2}-U_{s c f}\right)}\right)\right]
$$

Here, $K_{B}$ is the Boltzmann constant, $T$ is the Operating Temperature.

\section{III.RESULTS AND DISCUSSIONS}

CNT having a bandgap $\sim 0.83 \mathrm{eV}$, gate control parameter 0.88 and drain control parameter $\sim 0.035$ is used. To investigate the characteristics of the mentioned CNTFET, Fermi level is considered $\sim 0.32 \mathrm{eV}$. Different materials of various range of dielectric constant as oxide material has been taken within 4 to $120 . \mathrm{ON}$-state to OFF-state current ratio is analysed considering a fixed gate oxide thickness of $4 \mathrm{~nm}$ and different dielectric constant.

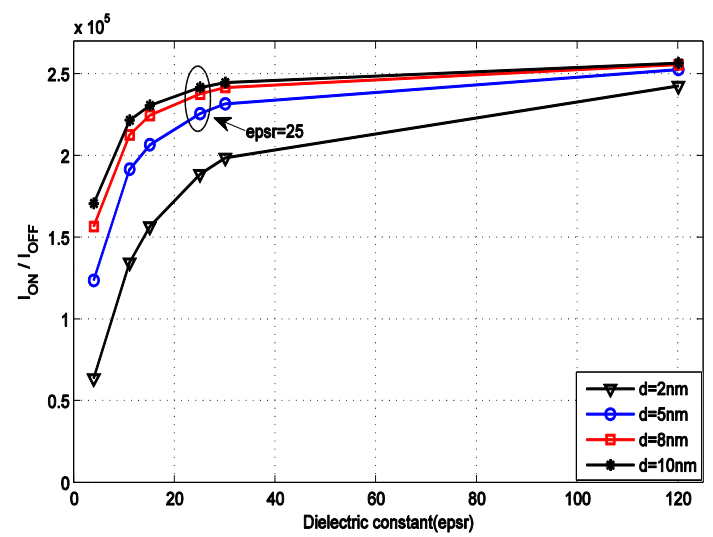

Fig. 2. ON-state to OFF-state current ratio vs Dielectric constant different tube diameter.

Fig. 2 shows the variation in ON-state to OFF-state current ratio with variable dielectric constant tube diameter. It is clear that there is a direct proportionality between dielectric constant and $\mathrm{ON}$-state to OFF-state current ratio. As much as we increase dielectric constant, the current ratio increases. But after a certain value of dielectric constant current ratio seems to be unchanged. It is seen that while varying dielectric constants, the change in $\mathrm{ON}$ state to OFF-state current ratios very much negligible as the change is below $5 \%$ while considering any material as gate oxide of dielectric constant 25 or above.

The above figure also indicates direct proportionality of tube diameter and $\mathrm{ON}$-state to $\mathrm{OFF}$-state current ratio. With the increment of tube diameter, current ratio also increases. A range of tube diameter which limits the change in ON-state to OFF-state current ratio within certain lower percentage value has also been examined. The tube diameter range here is considered in between $5 \mathrm{~nm}$ to $10 \mathrm{~nm}$. Within this range, ON-state to OFF-state current ratio remains unchanged.

To minimize the effect of change in gate oxide thickness on ON-OFF current ratio, effect of varying oxide thickness has been analyzed and an inverse relationship has been found. Increase in oxide thickness decreases the ON-state to OFF-state current Ratio.

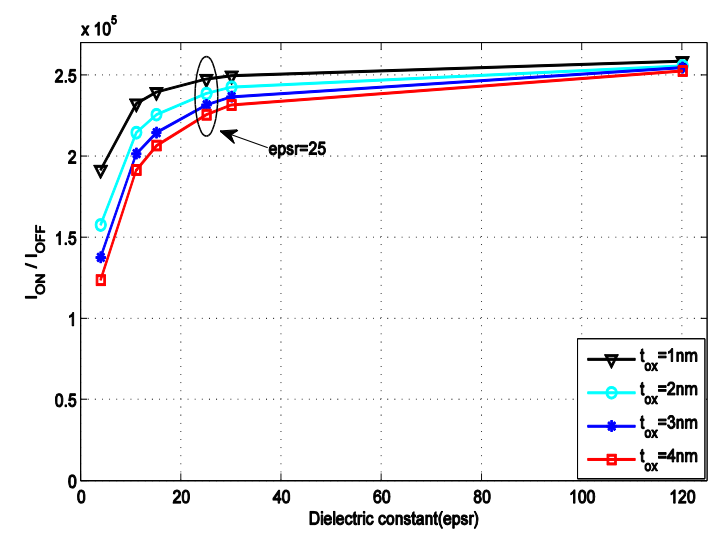

Fig. 3(a). ON-state to OFF-state current ratio vs Dielectric constant for gate oxide thickness $1 \mathrm{~nm}-4 \mathrm{~nm}$ 


\section{International Journal of Advanced Research in Computer and Communication Engineering}

Vol. 5, Issue 1, January 2016

Fig. 3(a) illustrates that increase in gate oxide thickness tube diameter decreases the current ratio which also decreases current ratio till a certain value of dielectric becomes almost unchanged within a certain range. Finally constant. If any material of dielectric constant above or we can conclude that any material having a dielectric equal 25 is chosen, then variation in oxide thickness has constant of 25 or above and a tube diameter of $5 \mathrm{~nm}$ or insignificant impact on ON-OFF current ratio. Further more makes the CNTFETs output current ratio almost simulation steps are taken to observe the outcome of the invariable. That is why an optimum combination of CNTFET at higher values of gate oxide thickness.

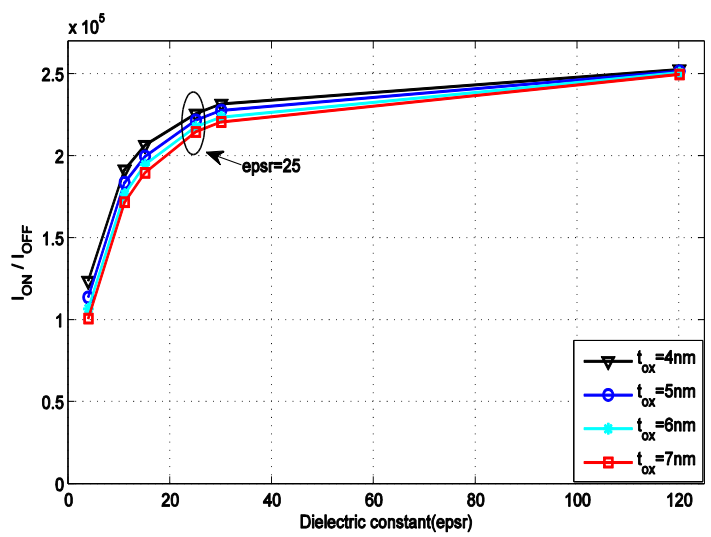

dielectric constant, tube diameter and gate oxide thickness should be chosen for designing purpose.

\section{REFERENCES}

[1] P. Avouris and J. Chen, "Nanotube electronics andoptoelectronics," Materials Today, vol. 9, no. 10,pp.46-54,2006.

[2] S. Iijima, "Helical microtubules of graphitic carbon", Nature, vol. 354, no. 6348, pp. 56-58, 1991.

[3] Arefinia Z., Orouji A.A.: 'Investigation of the novel attributesof a carbon nanotube FET withhigh-k gate dielectrics', Phys.E, 2008, 40, pp. 3068-3071.

[4] Hashim A.M., Ping H.H., Pin C.Y.: 'Characterization ofMOSFETlike carbon nanotube field effect transistor'.American Institute of Physics, ICAMN, 2010, 1217, (1), pp.11-17.

[5] Naderi A., Noorbakhsh M.S., Elahipanah H.: 'Temperaturedependence of electrical characteristics of carbon nanotubefield-effect transistors: a quantum simulation study', J. Nanomater.,2012,2012.

Fig. 3(b): ON-state to OFF-state current ratio vs Dielectric constant for gate oxide thickness $4 \mathrm{~nm}$ to above.

Fig. 3(b) demonstrates the plot of ON-state to OFF-state current ratio vs dielectric constant while higher values of oxide thickness ( $4 \mathrm{~nm}$ to $7 \mathrm{~nm}$ ) have been taken. The impact is minimized more keeping the current ratio almost unchanged.

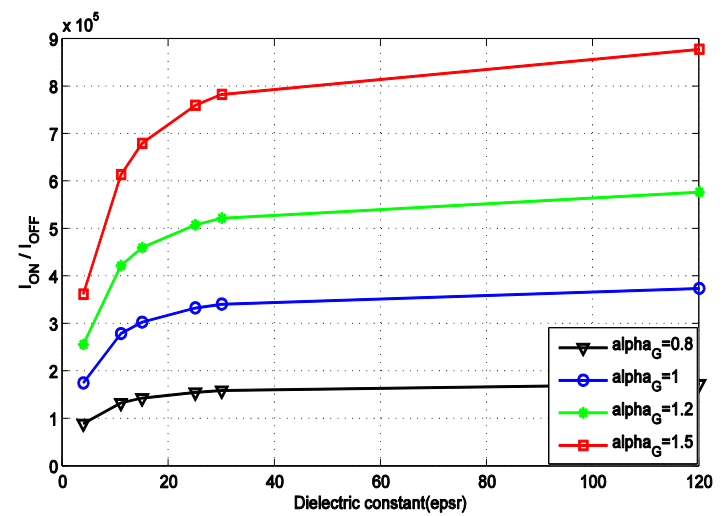

[6] Natori K.: 'Characteristics of carbon nanotube field effect transistor analyzed as a ballistic nano-wire field effect transistor', J. Appl. Phys., 2005, 97, (3), pp. 240-258.

[7] Rouf, NirjhorTahmidur; Deep, AshfaqulHaq; Hassan,RusafaBinte; Khan, Sabbir Ahmed; Hasan, Mahmudul;Mominuzzaman, Sharif Mohammad: 'High dielectric materialdependence of carbon nanotube field effect transistorconsidering non-ballistic conduction', Micro \&Nano Letters, 2014, 9, (10), p. 620-625.

[8] Safayat-Al Imam, Nasheen Kalam, Sharmin Abdhullah, 'Temperature Dependence of Carbon Nanotube FieldEffect Transistor under Non-Ballistic ConductionConsidering Different Dielectric Materials', Nanoscience and Nanotechnology 2014, 4(3), pp. 52-58.

[9] J Ali Naderi, S. Mohammad Noorbakhsh, and Hossein Elahipanah, "Temperature Dependence of Electrical Characteristics of Carbon Nanotube Field-Effect Transistors: A Quantum Simulation Study," Journal of Nanomaterials, vol. 2012, Article ID 532625,n7npages, 2012.doi:10.1155/2012/532625.

[10] P A Alvi et al., "Carbon nanotubes field effect transistors: A review," Indian Journal of Pure \& Applied physics, Vol.43, December, 2005

Fig 4. ON-state to OFF-state current ratio vs Dielectric constant for different gate control parameter

Fig. 4 states that increase in gate control parameter increases current ratio drastically. For the same dielectric constant this increase is very much significant. But as we can see, this impact is much lower if we choose lower values of dielectric constant. But in this case, other parameter such as dielectric constant does not maintain the desired range and is very much lower than we expected before.

\section{IV.CONCLUSION}

In this paper, by varying dielectric constant, tube diameter and gate oxide thickness, the performance of carbon nanotube field-effect transistors (CNTFETs) has been investigated. Increase in dielectric constant and tube diameter increases current ratio but after a certain value, this increment is not that much significant. Increase in 